\title{
Critical appraisal of the clinical practice guideline for the management of dyslipidaemia and prevention of cardiovascular disease: AACE 2017 guidelines.
}

\author{
ALKHAWAJA, R.M., MADI, L., ALHAMOUD, E., ABDALLAH, I., JAVED, M., \\ FAYEZ, H. and AL-ADAWI, R.M.
}

This is the peer reviewed version of the following article: ALKHAWAJA, R.M., MADI, L., ALHAMOUD, E., ABDALLAH, I., JAVED, M., FAYEZ, H. and AL-ADAWI, R.M. 2019. Critical appraisal of the clinical practice guideline for the management of dyslipidaemia and prevention of cardiovascular disease: AACE 2017 guidelines. Journal of pharmaceutical health services research, 10(3), pages 383-388, which has been published in final form at https://doi.org/10.1111/jphs.12302. This article may be used for non-commercial purposes in accordance with Wiley Terms and Conditions for Use of Self-Archived Version. 


\section{Critical appraisal of the clinical practice guideline for the management of dyslipidaemia and prevention of cardiovascular disease: AACE 2017 guidelines.}

Raja M. Alkhawajaa , Lama Madib, Eman Alhamoud ${ }^{a}$, Ibtihal Abdallaha, Mehak Javed ${ }^{a}$, Hamis Fayez ${ }^{a}$ and Rana Moustafa Al-Adawia

apharmacy Department, Hamad General Hospital (HGH) and bPharmacy Department, Qatar Rehabilitation Institute (QRI), HAMAD Medical Corporation (HMC), Doha, Qatar

Correspondence: Raja M. Alkhawaja, Pharmacy Department, Hamad General Hospital(HGH), HAMAD Medical Corporation (HMC), P.O Box 3050, Doha, Qatar.

E-mail: ralkhawaja@hamad.qa

\section{Abstract}

Objective: This study aims to appraise 2017 AACE Guidelines for Management of Dyslipidemia and Prevention of Cardiovascular Disease by using Appraisal of Guidelines for Research and Evaluation II (AGREE II) tool.

Method: A total of seven investigators who have obtained a postgraduate Doctor of Pharmacy or Masters of Clinical Pharmacy, appraised the dyslipidaemia guidelines independently, by using AGREE II tool.

Key findings: Among all the domains, the highest-scoring domain was the clarity of presentation $(87 \%)$, and the lowest was the applicability (26\%). The assessors gave the top ranking for both 'scope and purpose' (78\%) and 'Editorial independence' (79\%). The overall guideline assessment was $61 \%$. Most of the investigators (four out of seven) recommended using the guidelines in clinical practice with modifications.

Conclusion: The appraisal obtained in this article can be utilized by guideline developers to improve the quality of their upcoming guidelines. Healthcare professionals can be aware of guideline limitations and the importance of quality assessment of the guideline before applying their recommendations whenever possible by using Agree II tool.

Keywords: health services research; international; management 


\section{Background}

'Clinical Practice Guidelines', are defined by the Institute of Medicine as, 'Statements that include recommendations, intended to optimize patient care, that are informed by a systematic review of evidence and an assessment of the benefits and harms of alternative care options'. ${ }^{[1]}$ Historically, the Clinical Guidelines were designed to improve the quality and safety of health care. ${ }^{[2]}$ They have mainly focused on the size of effectiveness and the cost of interventions as well as the feasibility of applying the guidelines; however, recently 'Patient-Specific factors' are also incorporated to guide the treatment decisions, improve the quality and reduce the cost. ${ }^{[2]}$ Many standard methodologies and development strategies have been established to enhance the quality of new or updated guidelines; however, the adherence of the practice guidelines to the standards and development strategies is poor. ${ }^{[3]}$ Many key points are often not considered in developing guidelines, For example, 'Data Collection', 'Method Given' and 'Quality of Evidence Rated'. Moreover, the differences in opinions among guideline developers were not aired in guidelines and benefits of recommendations was given greater attention than potential harms. ${ }^{[4,5]}$

As mentioned, the appraisals of guidelines are significantly important. We aimed to critically appraise the 'Guidelines for management of Dyslipidemia and prevention of cardiology disease'. The 2017 AACE guideline which contains a total of 87 recommendations for a broader range of disease stages that include a new group of patients at risk and recommends more intense treatment of dyslipidaemia that recalls back LDL cholesterol targets? ${ }^{[6]}$

\section{Method}

The appraisal of this guideline was done by using the Appraisal of Guidelines for Research and Evaluation II (AGREE II) instrument. ${ }^{[7]}$ It is the only tool to assess the Table 1 Items and domains of the Appraisal of Guidelines for Research and Evaluation II (AGREE II) instrument quality of any clinical practice guidelines in any diseased area that can be used by healthcare providers, guideline developers, policy makers and educators'. It was published in 2003 and refined in 2009. The AGREE II tool to be validated requires the appraisal of six domains that involve 23 ranking items with additional categories for 'Overall Assessment' and 'recommendations' that scored with a 7-point scale. The domains include the following: scope and purpose, stakeholder involvement, rigour of development, clarity, and presentation, applicability and editorial independence. The largest number of key items is in the rigour of development section Table 1. ${ }^{[8]}$ AGREE II tool manual is available to explain in detail the subsections of each domain. ${ }^{[6]}$ The appraisers were asked to assign a grade between 1 (strongly disagree) and 7(strongly agree) for all the items, independently. At least two assessors (preferably four) are needed to appraise the guideline. Domain scores are calculated by summing up all the scores of the individual items in a domain and by scaling the total as a percentage of the maximum possible score for that domain Figure $1 .{ }^{[6]}$ A total of seven investigators either a postgraduate Doctor of Pharmacy or Masters of Clinical Pharmacy appraised the dyslipidaemia guideline independently; then, they forwarded their individually scored tool to one investigator (R.M.A), who reviewed and made sure that no item had scores differently by six points or more between assessors. If this conflict happened, the appraisal was distributed back to re-evaluate the ranking of a discrepant item. 


\section{Results}

Among all domains, the highest-scoring domain was the clarity of presentation (87\%), and the lowest was the applicability (26\%). The assessors gave the top ranking for both 'scope and purpose' $78 \%$ and 'Editorial independence' $79 \%$. The scoring for the others domain was as such: 'Stakeholder involvement' 56\%; 'Rigour of development' 35\%; 'Overall guideline assessment' $61 \%$. Most of the investigators (four out of seven) recommended using the guideline in clinical practice with modifications. The summary of appraisal results is shown in Table 2.

\section{Discussions}

The 'clarity of presentation' which is the highest ranked domain in our study is an essential aspect of using guidelines in practice; however, it does not indicate the methodological strength of guidelines. Moreover, it was reported as the highest ranking domain in many previous published reports. ${ }^{[9-11]}$

The applicability should be weighted more heavily through identifying the types of facilitators, barriers and advice to the clinician according to their settings. Although the applicability score was the lowest, most of the assessors agreed to use the guidelines in practice due to the availability of tools, resources, monitoring and follow-up criteria that facilitate its application. For dyslipidaemia guidelines, the rigour of development which describes the methodology, consisting of seven items was scored (35\%). This can be enhanced clearly by explaining the criteria for searching and selecting the evidence, providing a procedure for updating the guidelines and considering the health benefits, side effects and harms when formulating the recommendation. The low score for stakeholder involvement $(56 \%)$ in dyslipidemia guideline was due to the lack of information about the intended user experience and views of healthcare professionals in the development process of the guidelines. More so, this information if included during the process of development of guidelines may enhance patient understanding and compliance.

\section{Conclusion}

In conclusion, the appraisal obtained in this article can be utilized by guideline developers to improve the quality of their upcoming guidelines. Healthcare professionals can be aware of guideline limitations and the importance of quality assessment of the guideline before applying their recommendations whenever possible by using Agree II tool. 
Table 1 - Items and domains of the Appraisal of Guidelines for Research and Evaluation II (AGREE II) instrument.

Item Content

1 The overall objective(s) of the guideline is (are specifically described)

2 The health question(s) covered by the guideline is (are specifically described)

3 The population (patients public etc.) to whom the guideline is meant to apply is specifically described

$4 \quad$ The guideline development group includes individuals from all relevant professional groups

5 The views and preferences of the target population (patients public etc.) have been sought

6 The target users of the guideline are clearly defined

7 Systematic methods were used to search for evidence

8 The criteria for selecting the evidence are clearly described

9 The strengths and limitations of the body of evidence are clearly described

10 The methods for formatting the recommendations are clearly described

11 The health benefits, side effects and risks have been considered in formulating the recommendations

12 There is an explicit link between the recommendations and the supporting evidence

13 The guideline has been extremely reviewed by experts prior to its publication

14 A procedure for updating the guideline is provided

15 The recommendations are specific and unambiguous

16 The different options for management of the condition or health issue are clearly presented

17 Key recommendations are easily identifiable

18 The guideline describes facilitators barriers to its application

19 The guideline provides advice and/or tools on how the recommendations can be put into practice

20 The potential resource implications of applying the recommendations have been considered

21 The guideline presents monitoring and/or auditing criteria

22 The views of the funding body have not influenced the content of the guideline

23 Competing interest of guideline development group members have been recorded and addressed
Domain

Scope and purpose

Stakeholder involvement

Rigour of development

Clarity of presentation

Applicability

Editorial independence

Extracted from the AGREE II instrument. 
A quality score is calculated for each of the six AGREE II domains. The six domain scores are independent and should not be aggregated into a single quality score.

\section{Calculating Domain Scores}

Domain scores are calculated by summing up all the scores of the individual items in a domain and by scaling the total as a percentage of the maximum possible score for that domain.

\section{Example:}

If 4 appraisers give the following scores for Domain 1 (Scope \& Purpose):

\begin{tabular}{lcccc} 
& Item 1 & Item 2 & Item 3 & Total \\
\hline AppraIser 1 & 5 & 6 & 6 & $\mathbf{1 7}$ \\
Appraiser 2 & 6 & 6 & 7 & $\mathbf{1 9}$ \\
Appraiser 3 & 2 & 4 & 3 & $\mathbf{9}$ \\
Appraiser 4 & 3 & 3 & 2 & $\mathbf{8}$ \\
\hline Total & $\mathbf{1 6}$ & $\mathbf{1 9}$ & $\mathbf{1 8}$ & $\mathbf{5 3}$
\end{tabular}

Figure 1 Calculating \& scoring the Appraisal of Guidelines for Research and Evaluation II (AGREE II).

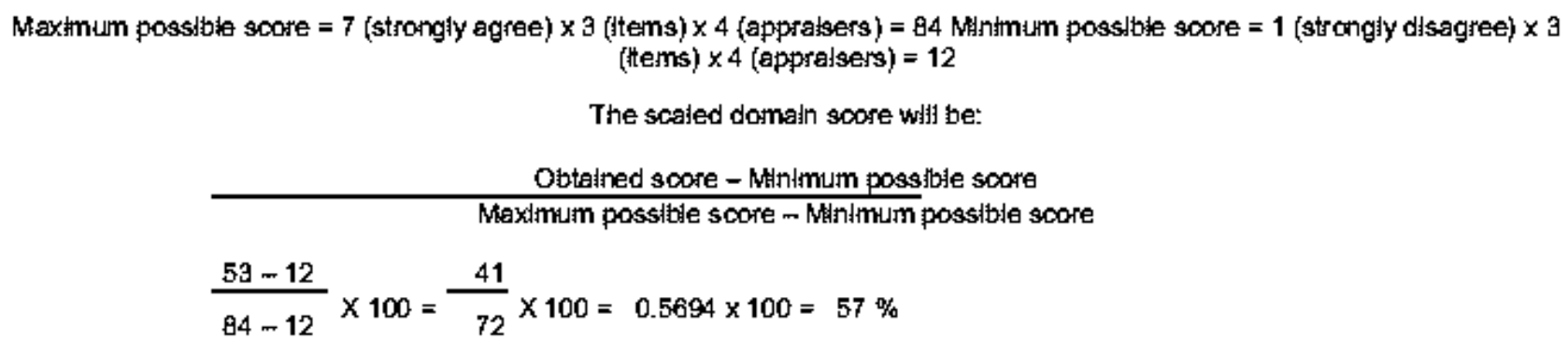


Table 2 Summary of appraisal results

\begin{tabular}{lc}
\hline Domain & Scaled domain score (\%) $^{\dagger}$ \\
\hline Scope and purpose & 78 \\
Stakeholder involvement & 56 \\
Rigour of development & 35 \\
Clarity of presentation & 87 \\
Applicability & 26 \\
Editorial independence & 79 \\
Overall guideline assessment & 61 \\
Overall guideline recommendation & Yes, with modification \\
\hline
\end{tabular}

${ }^{\dagger}$ Based on scoring (on a 7-point Likert scale) by seven assessors, with standardized domain scores subsequently calculated according to AGREE II formula and reported as percentages (highest possible score: $100 \%$ ).

\section{Declarations}

\section{Conflict of interest}

The authors declare that they have no conflicts of interest to disclose.

\section{Funding}

This research received no specific grant from any funding agency in the public, commercial or not-for-profit sectors.

\section{Authors' contributions}

All authors were involved in appraising the guidelines. RMA designed the study, collected and analyzed the data. RMA, LM wrote the drafted paper. RMA, LM, MJ reviewed and finalized it.

\section{References}

1 Consensus Report, Institute of Medicine. Clinical Practice Guidelines We Can Trust. March 23, 2011. http://www.iom.edu/Reports/2011/Clinical-Practice-Guidelines-WeCan-Trust.aspx (accessed 15 September 2017).

2 Eddy DM et al. Individualized guidelines: the potential for increasing quality and reducing costs. Ann Intern Med 2011; 154: 627.

3 Burgers J et al. Clinical practice guidelines as a tool for improving patient care. In: Grol $\mathrm{R}$ et al., eds. Improving Patient Care. The Implementation of Change in Clinical Practice. Hoboken, NJ: John Wiley \& Sons, 2013: 91-114.

$4 \quad$ Kung $\mathrm{J}$ et al. Failure of clinical practice guidelines to meet institute of medicine standards two more decades of little, if any, progress. Arch Intern Med 2012; 172: 1628-1633.

5 Shaneyfelt TM et al. Are guidelines following guidelines? The methodological quality of clinical practice guidelines in the peer-reviewed medical literature. JAMA 1999; 281: 1900. 
Jellinger PS et al. American Association of Clinical Endocrinologists and American College of Endocrinology Guidelines for management of dyslipidemia and prevention of cardiovascular disease. Endocr Pract 2017; 2: 1-87.

7 Brouwers $M$ et al. AGREE Next Steps Consortium AGREE II: advancing guideline development, reporting and evaluation in healthcare. Can Med Assoc J 2010; 182: e839-e842.

8 Hoffmann-Eßer W et al. Guideline appraisal with AGREE II: systematic review of the current evidence on how users handle the 2 overall assessments. PLoS One 2017; 12: e0174831.

9 Gorman SK et al. A critical appraisal of the quality of critical care pharmacotherapy clinical practice guidelines and their strength of recommendations. Intensive Care Med 2010; 36: 1636.

10 Farghali AA et al. Rigorous method to assess quality and generalizability of clinical practice guidelines. Can J Hosp Pharm 2014; 67: 397-398.

11 Wilby $\mathrm{KJ}$ et al. Critical appraisal of clinical practice guidelines in pediatric infectious disease. Int J Clin Pharm 2015; 37: 799. 